\title{
BMJ Open Incidence of laboratory-confirmed influenza disease among infants under 6 months of age: a systematic review
}

\author{
Deshayne B Fell, ${ }^{1}$ Jeanene Johnson, ${ }^{2}$ Zohar Mor, ${ }^{3,4}$ Mark A Katz, ${ }^{5,6}$ \\ Becky Skidmore, ${ }^{7}$ Kathleen M Neuzil, ${ }^{8}$ Justin R Ortiz, ${ }^{9}$ Niranjan Bhat ${ }^{10}$
}

To cite: Fell DB, Johnson J, Mor Z, et al. Incidence of laboratory-confirmed influenza disease among infants under 6 months of age: a systematic review. BMJ Open 2017;7:e016526. doi:10.1136/ bmjopen-2017-016526

- Prepublication history and additional material for this paper are available online. To view these files please visit the journal online (http://dx.doi. org/10.1136/bmjopen-2017016526).

Received 22 February 2017

Revised 2 June 2017

Accepted 12 June 2017

\section{CrossMark}

For numbered affiliations see end of article.

Correspondence to

Dr Niranjan Bhat;

nbhat@path.org

\section{ABSTRACT}

Objectives The aim of this systematic review was to assess incidence rates of laboratory-confirmed influenza (LCl) outcomes among infants under 6 months of age. Design Systematic literature search and review of indexed studies in PubMed, EMBASE, the Cochrane Library and CINAHL Plus from inception to 19 April 2017.

Setting Population-based estimates from community or hospital settings.

Participants Infants under 6 months of age.

Primary and secondary outcome measures $\mathrm{LCl}$ illness in ambulatory care settings, $\mathrm{LCl}$ hospitalisation, $\mathrm{LCl}$ intensive care unit admission and LCl death. Only studies with population-based incidence data were included.

Results We identified 27 primary studies, 11 of which were from the USA, four were from other non-US highincome settings and the remaining were from lowermiddle-income or upper-middle-income countries. Most studies ( $n=23$ ) assessed incidence of $\mathrm{LCl}$ hospitalisation, but meta-analysis to pool study-specific rates was not possible due to high statistical and methodological heterogeneity. Among US studies, the reported incidence of $\mathrm{LCl}$ hospitalisation ranged from 9.3 to 91.2 per 10000 infants under 6 months for seasonal influenza, while the only US-based estimate for pandemic H1N1 influenza was 20.2 per 10000 infants. Reported rates for LCI hospitalisation for seasonal influenza from other countries ranged from 6.2 to 73.0 per 10000 infants under 6 months, with the exception of one study with an estimated rate of 250 per 10000 infants. No events were reported in five of the nine studies that evaluated $\mathrm{LCl}$ death among infants under 6 months.

Conclusion Our review of published studies found limited data on LCl outcomes for infants under 6 months, particularly from non-US settings. Globally representative and reliable incidence data are necessary to fully evaluate influenza disease burden and the potential impact of maternal influenza immunisation programme on morbidity and mortality in young infants.

\section{INTRODUCTION}

The influenza virus is a common pathogen identified in young children with acute lower respiratory infections, such as pneumonia and bronchiolitis, ${ }^{1}$ globally accounting for approximately $10 \%$ of all respiratory hospitalisations in children under 18 years $^{2}$ and

\section{Strengths and limitations of this study}

This review of laboratory-confirmed, populationbased estimates of influenza incidence highlights the relative lack of studies that specifically report influenza outcomes among infants under the age of 6 months.

- The majority of data identified in this review originate from the USA, deriving primarily from just two influenza surveillance systems, posing challenges for estimating the impact of maternal influenza immunisation programmes on infant influenza outcomes, particularly for low-income and middle-income countries.

- We were unable to perform any meta-analyses due to high methodological and statistical heterogeneity.

approximately $3 \%$ of postneonatal deaths. ${ }^{3}$ Influenza virus infection can also manifest in various other conditions including seizures, wheezing, croup, otitis media and occasionally encephalitis and encephalopathy, ${ }^{4-7}$ and it can progress to secondary bacterial pneumonias or exacerbate underlying chronic medical conditions.

Infants under 6 months of age are considered to be at high risk for severe influenza and associated complications due to documented high rates of influenza-associated hospitalisation $^{8-12}$ and mortality. ${ }^{7}$ However, since influenza vaccines are not licensed for use in this age group due to poor immunogenic responses to the vaccine, ${ }^{13}$ protection of newborns and young infants from influenza virus infection and related complications requires alternate strategies. ${ }^{14}$ One such strategy is immunisation of pregnant women with influenza vaccine, which has been shown to reduce influenza virus infection among young infants through transplacental transfer of maternal anti-influenza antibodies. ${ }^{15-17}$

A 2011 systematic review and meta-analysis by Nair et $a l^{1}$ estimated the global incidence of influenza outcomes among children under the age of 5 years and concluded that 
influenza in young children results in significant utilisation of health services, particularly among infants younger than 1 year. However, age-specific estimates for infants under 6 months were not reported. ${ }^{1}$ There is a paucity of published data on incidence of influenza outcomes among children in this younger age group,${ }^{14}$ yet these data are necessary for informing evidence-based decision making regarding vaccination programmes, provision of appropriate health services and prioritising future research. In 2014, the WHO formed a working group ${ }^{18}$ to systematically review the evidence and estimate incidence rates of laboratory-confirmed influenza (LCI) outcomes among infants less than 6 months of age.

\section{METHODS}

In consultation with the WHO influenza working group, ${ }^{18}$ we developed a systematic review protocol (available on request). This manuscript was prepared following the Preferred Reporting Items for Systematic Reviews and Meta-Analyses recommendations. ${ }^{19}$

\section{Search strategy and study selection}

Our search strategy was developed by an experienced medical information specialist based on the review protocol (available from author on request) and informed by the approach used by Nair et al in their systematic review of the global influenza burden among young children. ${ }^{1}$ We searched PubMed, EMBASE, the Cochrane Library and CINAHL Plus with Full Text from inception to 19 April 2017. Our strategies used a combination of controlled vocabulary (eg, 'Influenza, Human', 'Infant Mortality' and 'Incidence') and keywords (eg, influenza, neonate and rate). No language or date restrictions were applied but animal-only and opinion pieces (eg, comments, editorials and interviews) were removed from the initial search results where possible. No unpublished data were pursued or included. Specific details regarding the search strategies appear in online supplementary appendix 1 .

We used Reference Manager V.12 to download our search results and remove duplicates. Abstracts were then exported to Abstrackr (http://abstrackr.cebm. brown.edu/) for screening and data abstraction. Two teams of two reviewers independently screened all titles and abstracts to identify potentially relevant articles for full-text review. Disagreements between reviewers were resolved through discussion and consensus. The same two teams of reviewers carried out full-text screening to identify studies that met all the inclusion and exclusion criteria for data extraction and quality assessment. While extracting data, the reviewers also examined the reference lists to identify potentially relevant articles that may have been missed during screening.

We included studies that reported original data on population-based incidence rates for the following LCI outcomes among infants under 6 months of age: LCI illness in ambulatory care settings, LCI hospitalisation,
LCI intensive care unit (ICU) admission and LCI death. We excluded studies that did not ascertain LCI outcomes either throughout at least one full influenza season in geographic settings with defined seasonality, or otherwise for at least one full year. We also excluded studies if influenza was not examined as a primary outcome, but rather as a co-infection in a study population identified on the basis of another infectious disease (eg, influenza co-infection in a cohort of hospitalised measles cases ${ }^{20}$ ). Finally, studies that used a case definition that was not clearly defined or consistently applied, and those that were not population based or had a population denominator of fewer than 500 infants under 6 months of age were excluded. We included data from the comparator group of any randomised controlled trials (RCTs) on influenza immunisation during pregnancy if the study otherwise met our inclusion criteria.

\section{Data extraction and quality assessment}

Data from each included study were abstracted by one of two reviewers using a standardised data extraction form which was first pilot-tested to ensure a high level of agreement between reviewers. We extracted the following, where available, from each study: author; publication year; study design; study country; study population and size; age ranges studied; subject selection criteria; length of surveillance period and influenza season (particularly specifying the 2009-2010 pandemic vs other seasons); circulating influenza virus strains; definition and type of outcomes included in the study; methods for ascertaining cases (eg, active vs passive surveillance); criteria used for influenza testing; laboratory assay used to confirm influenza diagnosis; influenza vaccination coverage in pregnant women in locations where the studies were conducted. We also extracted information, where available, on the numerator and denominator for each incidence rate; any statistical analyses performed, including variables used to compute adjusted rates; crude and adjusted incidence rates for each outcome with 95\% CIs or other measures of variance; any sensitivity analyses presented in the paper. Study authors were contacted as needed to clarify data or methods. Two independent reviewers evaluated the quality of each study. Since all included studies were case series or surveillance studies that did not include comparative analyses, we used a modification of the Joanna Briggs Institute (JBI) Critical Appraisal Checklist for Descriptive/Case Series to assess individual study quality. ${ }^{21}$ This checklist assesses four items: clearly defined case inclusion criteria, objective assessments of exposure and outcome, and sufficient follow-up time for outcome ascertainment. In addition, we assessed the quality of evidence across studies using an adaptation of the Grading of Recommendations Assessment, Development and Evaluation (GRADE) framework. ${ }^{22}{ }^{23}$ For each outcome, we determined a GRADE rating of high quality, moderate quality, low quality or very low quality for each outcome according to criteria such as study design and limitations, inconsistency in study findings and imprecision. ${ }^{22} 23$ 


\section{Data synthesis and analysis}

We qualitatively summarised individual study characteristics in descriptive tables. For each outcome, we extracted the incidence rates and $95 \%$ CIs as reported by the primary study when they were provided, and otherwise computed them using raw study data where possible. We estimated the numerator or denominator values when an unadjusted incidence rate was reported along with only one of the other two data points. We interpreted incidence rates (computed using person-time denominators for infants under 6 months) and incidence proportions (computed using the estimated size of the population of infants under 6 months) as approximately equivalent. No attempt was made to mathematically convert one to the other since most studies that reported an incidence proportion used the total number of infants under the age of 1 year and divided in half, which would approximate 6 months of person-time follow-up assuming a static population with no losses to follow-up. We used Stata SE software V.12 (Stata-Corp LP) to generate pooled incidence estimates for LCI hospitalisation via random effects meta-analyses ${ }^{24}$ and the $\mathrm{I}^{2}$ statistic to quantitatively assess statistical heterogeneity. ${ }^{25}$ Pooled incidence estimates were not reported when statistical heterogeneity was high (ie, $\mathrm{I}^{2}>75 \%$ ); however, qualitatively, we explored sources of heterogeneity in subgroups to augment our interpretation. A priori, we hypothesised that heterogeneity would likely arise due to differences in pandemic versus seasonal influenza, study population, case ascertainment methods and study quality. We generated forest plots using the R package 'ggplot2' (R Foundation for Statistical Computing).

\section{RESULTS}

\section{Study selection}

We identified 9298 records through our initial electronic literature searches; following de-duplication, 5998 went through initial title and abstract screening. We identified 150 potentially relevant articles and excluded 125 after full-text review, leaving 25. Most manuscripts (81/125; $65 \%$ ) were excluded because they lacked age-specific data on infants under 6 months. During full-text screening, we added two articles that had originally been excluded by our systematic query but were subsequently identified through a hand search of reference lists. ${ }^{1626}$ This brought the total number of primary studies included in our review to 27 (figure 1).

\section{Study characteristics}

Nearly half $(11 / 27)$ of the studies originated from the $\mathrm{USA}^{7-9}{ }^{26-33}$ and the remaining were from lower-middle-income $\quad(n=5),{ }^{11}{ }^{17} \quad 34-36 \quad$ upper-middle-income $(\mathrm{n}=7)^{16 \quad 37-42}$ and other non-US high-income settings $(n=4)^{43-46}$ (table 1$)$. There were no studies from low-income countries. Twenty-three studies assessed LCI hospitalisation, 89111617 26-33 3536 38-41 43-46 six studies assessed LCI illness in ambulatory care settings, ${ }^{9} 1617343742$ seven studies reported LCI ICU admission 9263239404546 and nine assessed LCI deaths 71116172628354044 (table 1). All studies were published in 2004 or later and reported data from influenza seasons between 2000 and 2014. Two studies exclusively reported influenza outcomes from the 2009 H1N1 pandemic time period, ${ }^{40} 46$ six reported data from the 2009 pandemic time period along with other influenza seasons ${ }^{27} 35-374344$ and the remaining reported LCI outcomes from seasonal influenza epidemics. Most studies used reverse transcription (RT) PCR laboratory testing methods, either alone or in combination with other methods, to confirm influenza from patient samples (online supplementary table S1). Four of the 25 non-randomised trials included in this review 394144 provided some contextual information on uptake of maternal influenza immunisation in their study population, but none provided specific rates (online supplementary table S2). We were able to obtain additional clarifying data from four $^{16274042}$ of six studies by contacting study authors. Only two studies received a score lower than $4 / 4$ on the modified JBI Critical Appraisal Checklist-one such study did not assess all influenza outcomes using objective criteria $^{44}$ and the other did not clearly document the case definition. ${ }^{46}$ Applying the modified GRADE assessment, ${ }^{23}$ the quality of evidence for incidence rates of LCI hospitalisation of infants under 6 months of age was deemed to be moderate, while the quality of evidence for the other three outcomes was considered low (online supplementary appendix 2). In large part, this determination was based on high heterogeneity in incidence rates across studies, partly due to variability in surveillance methodologies and methods used to compute rates. Moreover, other than hospitalisation, the number of studies reporting data for the other outcomes of interest was small.

\section{LCI illness in ambulatory care settings}

Six studies, two of which were from lower-income or lower-middle-income countries, assessed LCI illness in ambulatory care settings ${ }^{9} 1617343742$ (table 2). Five of the studies used RT-PCR to confirm influenza virus infection, and one used either RT-PCR or viral culture ${ }^{9}$ (online supplementary table $\mathrm{S} 1$ ). In a community-based prospective cohort study conducted between 2009 and 2011 in the Cajamarca region of Peru, researchers conducted active household surveillance to identify infants with symptoms of acute respiratory illness for confirmatory influenza laboratory testing. In this study, the adjusted incidence of LCI illness among infants less than 6 months of age was 35 per 100 person-years of follow-up (95\% CI 26 to 48) ${ }^{37}$ Using similar active surveillance methods, the RCT from South Africa reported an incidence of LCI illness among infants born to non-HIV infected women in the placebo group of 3.6 per 100 infants (95\% C: 2.6 to 5.0), ${ }^{16}$ and the RCT from Mali reported an incidence of 8.3 per 100 person-years among infants in the control arm. ${ }^{17}$ In a study conducted over three influenza seasons in the Suzhou District of China, the incidence of LCI illness among infants under 6 months ranged from 2.3 per 100 


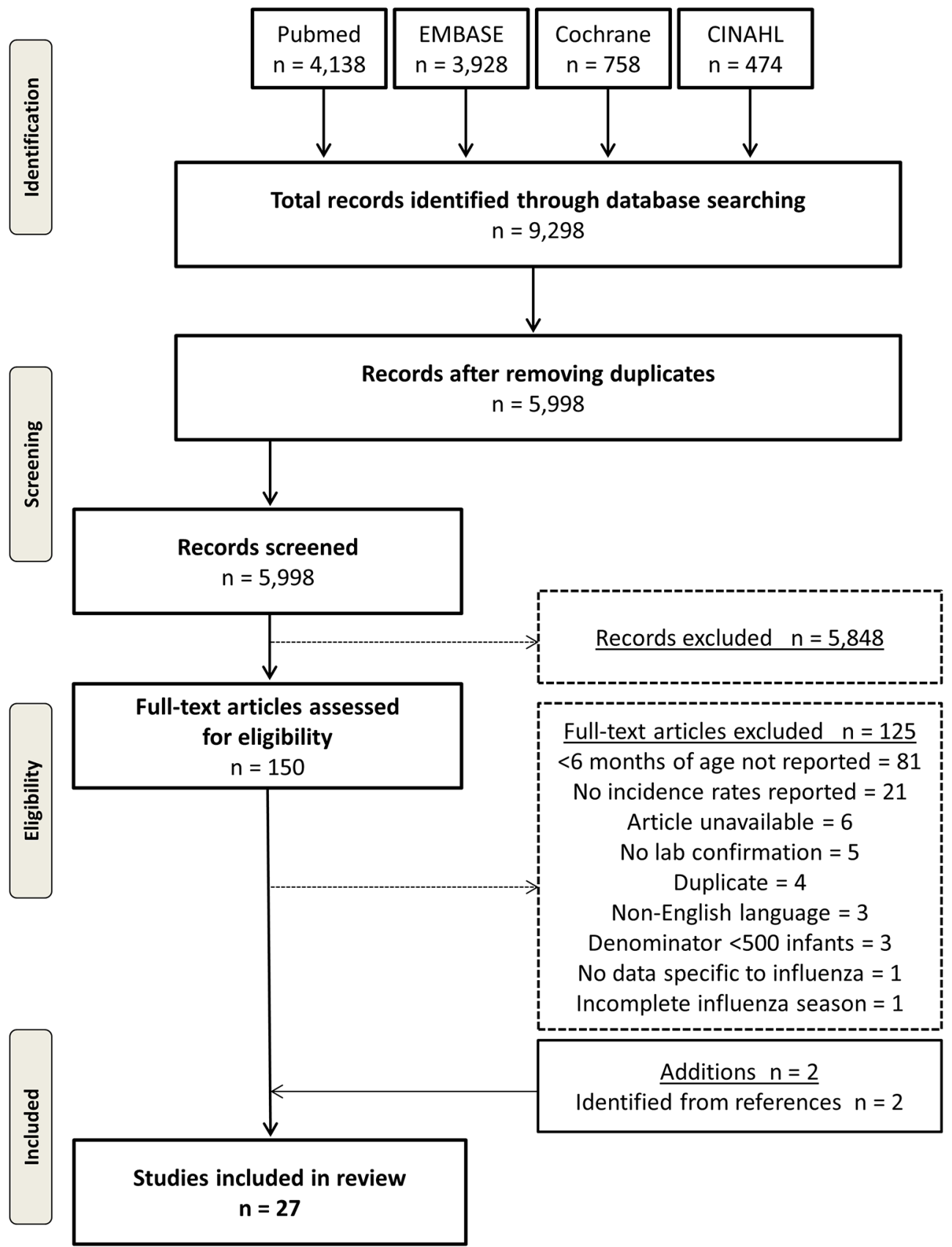

Figure 1 Preferred Reporting Items for Systematic Reviews and Meta-Analyses flow diagram showing selection of studies.

in 2013-2014 to 2.9 per 100 in 2012-2013. ${ }^{42}$ Finally, data from the New Vaccine Surveillance Network (NVSN), a population-based active sentinel surveillance programme operating in three regions of the USA (Davidson County, Tennessee; Hamilton County, Ohio; and Monroe County, New York), estimated a rate of LCI illness based on outpatient clinic visits among infants under 6 months of 2.8 per 100 infants (95\% CI 0.7 to 11.1 ) in 2002-2003 and 5.9 per 100 infants (95\% CI 2.8 to 12.8 ) in $2003-2004 .{ }^{9} \mathrm{We}$ did not consider statistical meta-analysis of this outcome due to the variable geographic settings and methodologies employed by the studies. For instance, the latter NVSN study estimated incidence rates from population-based surveillance of outpatient clinic visits, ${ }^{9}$ while the study from Peru used community-based surveillance including a household component, ${ }^{37}$ and the two RCTs employed active surveillance with weekly contact with study participants. ${ }^{1617}$

\section{LCI hospitalisation}

Graphical and tabular summaries of individual estimates originating from the 23 studies that reported incidence rates of LCI hospitalisation can be found in figure 2 and online supplementary table S3. Ten of the 23 studies originated from the USA; six of those reported data from one of two population-based active surveillance programme: the Emerging Infections Program (EIP) $)^{2728}$ or the NVSN. ${ }^{8929}$ Two additional US studies 


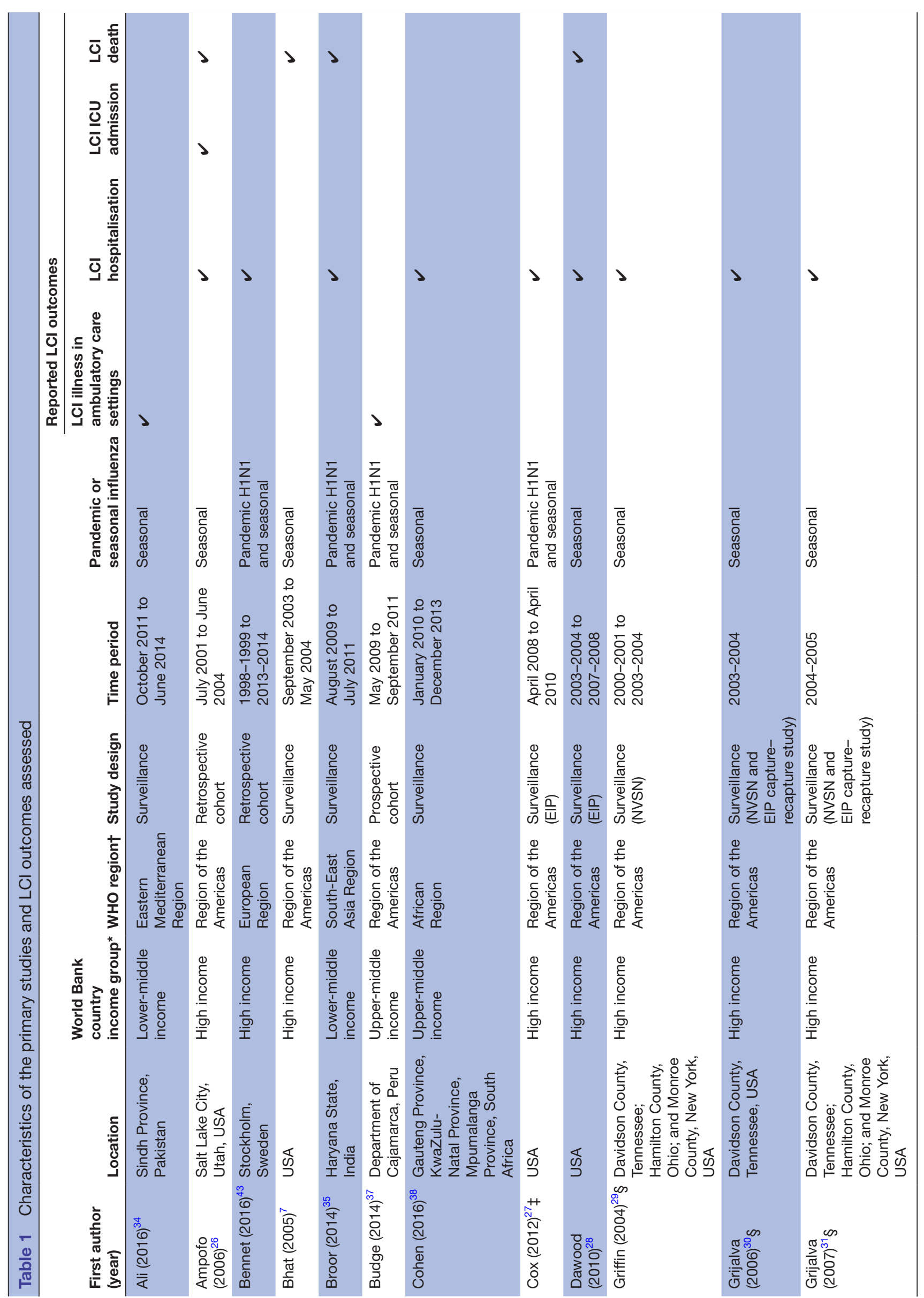




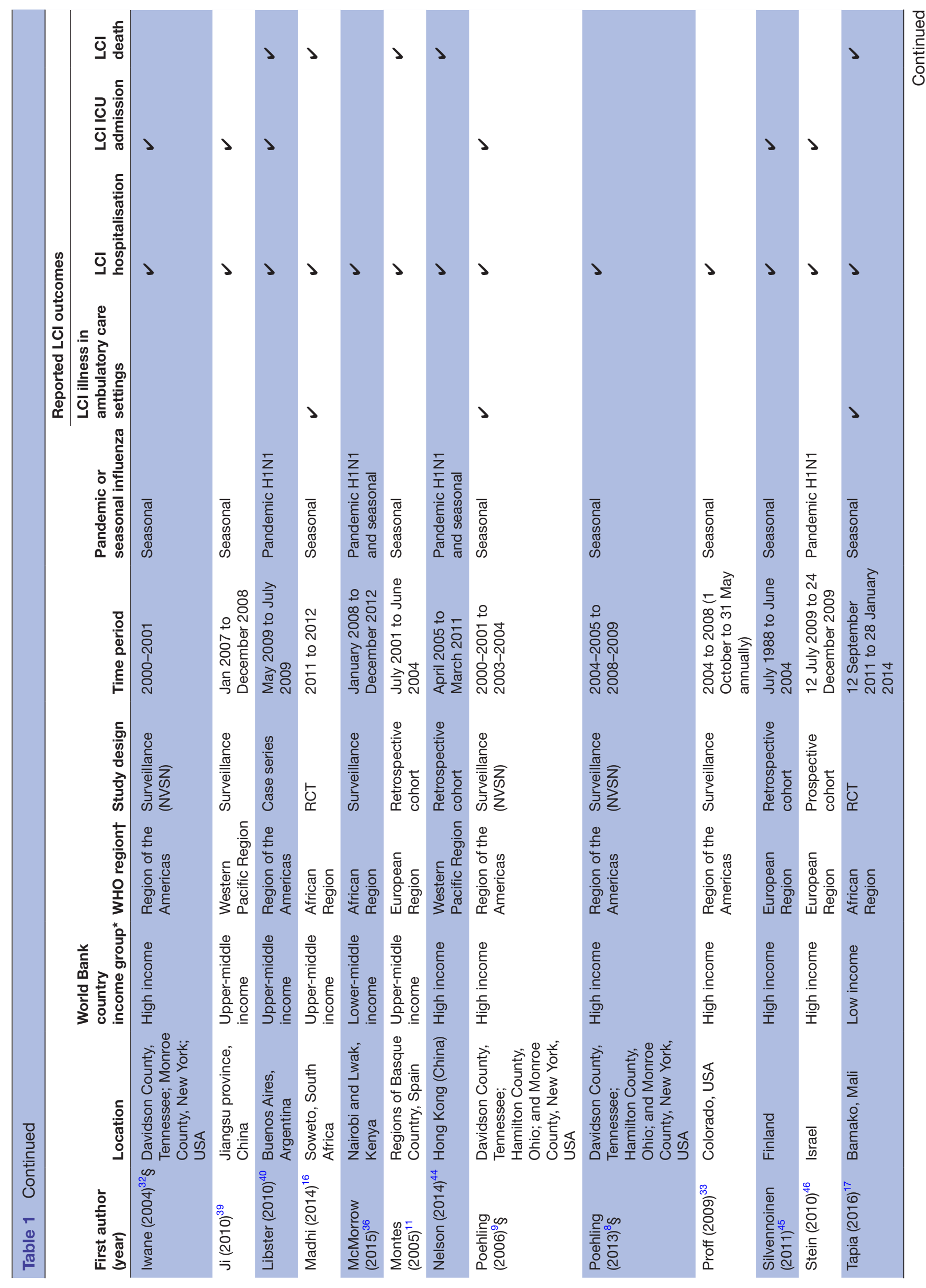




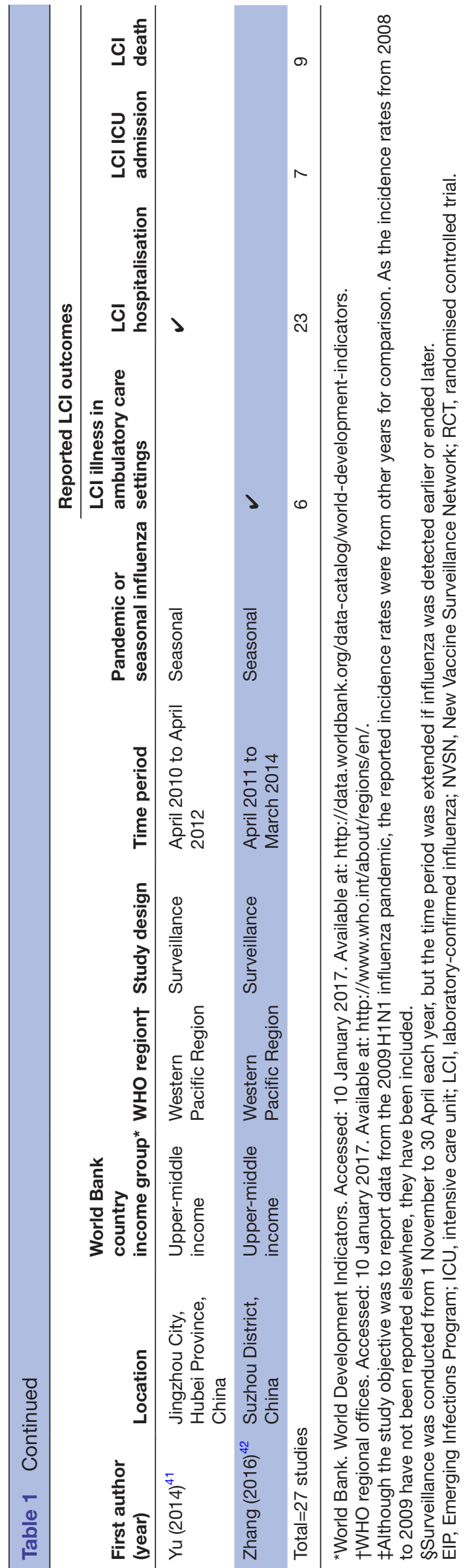

led by Grijalva et al reported estimates using capturerecapture methods based on surveillance data from both the EIP and NVSN systems, ${ }^{30}{ }^{31}$ and the remaining two US studies reported data from separate systems. ${ }^{26}{ }^{33}$ In the USA, estimated rates of LCI hospitalisation of infants less than 6 months of age during seasonal epidemics varied from a low of 9.3 per 10000 infants (95\% CI 7.9 to 10.9$)$ in $2006-2007^{28}$ to a high of 91.2 per 10000 infants $(95 \%$ CI 67 to 145$)^{30}$ in 2003-2004. The only US-based estimate for the 2009 pandemic H1N1 time period was 20.2 per 10000 infants (95\% CI 18.1 to 22.5$).{ }^{27}$

The 13 non-US studies (four from high-income countries, six from upper-middle-income countries, two from lower-middle-income countries and one from a low-income country) reported similar LCI hospitalisation rates for seasonal influenza. Most incidence rates for seasonal influenza ranged from 6.2 per 10000 infants (95\% CI 3.1 to 9.3) in China in $2007^{39}$ to 73.0 per 10000 infants $(95 \%$ CI 40.6 to 121.7) in Spain in 2003-2004. ${ }^{11}$ However, a higher estimated rate was reported from one postpandemic study of seasonal influenza from China (250 per 10000 infants under 6 months in 2010-2011, 95\% CI 213 to 292). ${ }^{41}$ The highest estimate from non-US based studies from the 2009 pandemic H1N1 influenza time period was 259 per 10000 person-years (95\% CI 97.0 to 689) in Kenya. ${ }^{36}$

Although 10 studies from the USA presented incidence rates of LCI hospitalisation of infants under 6 months, there was overlap in a number of seasons among the eight studies using data from the EIP and NVSN surveillance programmes (table 3). For instance, two studies reported the same rate from the NVSN system for the 2000-2001 season (24.0 per 10000 infants), ${ }^{932}$ and similar combined season rates for 2000-2004 from the NVSN system (reported as 43.0 per 10000 infants $^{29}$ and 45.0 per 10000 infants $^{9}$ ). In addition, estimates are available from both the EIP and the NVSN for several years. In such instances, incidence rates from the NVSN system were consistently higher in magnitude than the EIP estimates. Moreover, in two studies, Grijalva et al combined data from the EIP and NVSN surveillance systems using a capture-recapture methodology ${ }^{30}{ }^{31}$ - a surveillance method that attempts to estimate the extent of under-ascertainment of cases using information from two or more data sources. ${ }^{47}$ This methodology yielded a higher combined incidence rate of LCI hospitalisation than was provided by either system alone. ${ }^{30}$ For instance, in 2003-2004, which was a more severe influenza season, individual EIP and NVSN estimates were 29.6 per 10000 infants (95\% CI 26.7 to 32.8$)^{28}$ and 72.0 per 10000 infants (95\% CI 53.0 to 92.0$){ }^{9}$ respectively. Using the combined data from both systems, the revised estimate was 91.2 per 10000 infants (95\% CI 67.0 to 145.0$).{ }^{30}$

\section{LCI ICU admission}

LCI ICU admission rates for infants under 6 months are available from seven studies ${ }^{96} 3239404546$ (table 4). However, all rates shown in table 4 were computed by review authors, either due to non-reporting in the original study 92228333437 or due to graphical presentation of rates 
Table 2 Incidence estimates of laboratory-confirmed influenza illness in ambulatory care settings among infants under 6 months of age

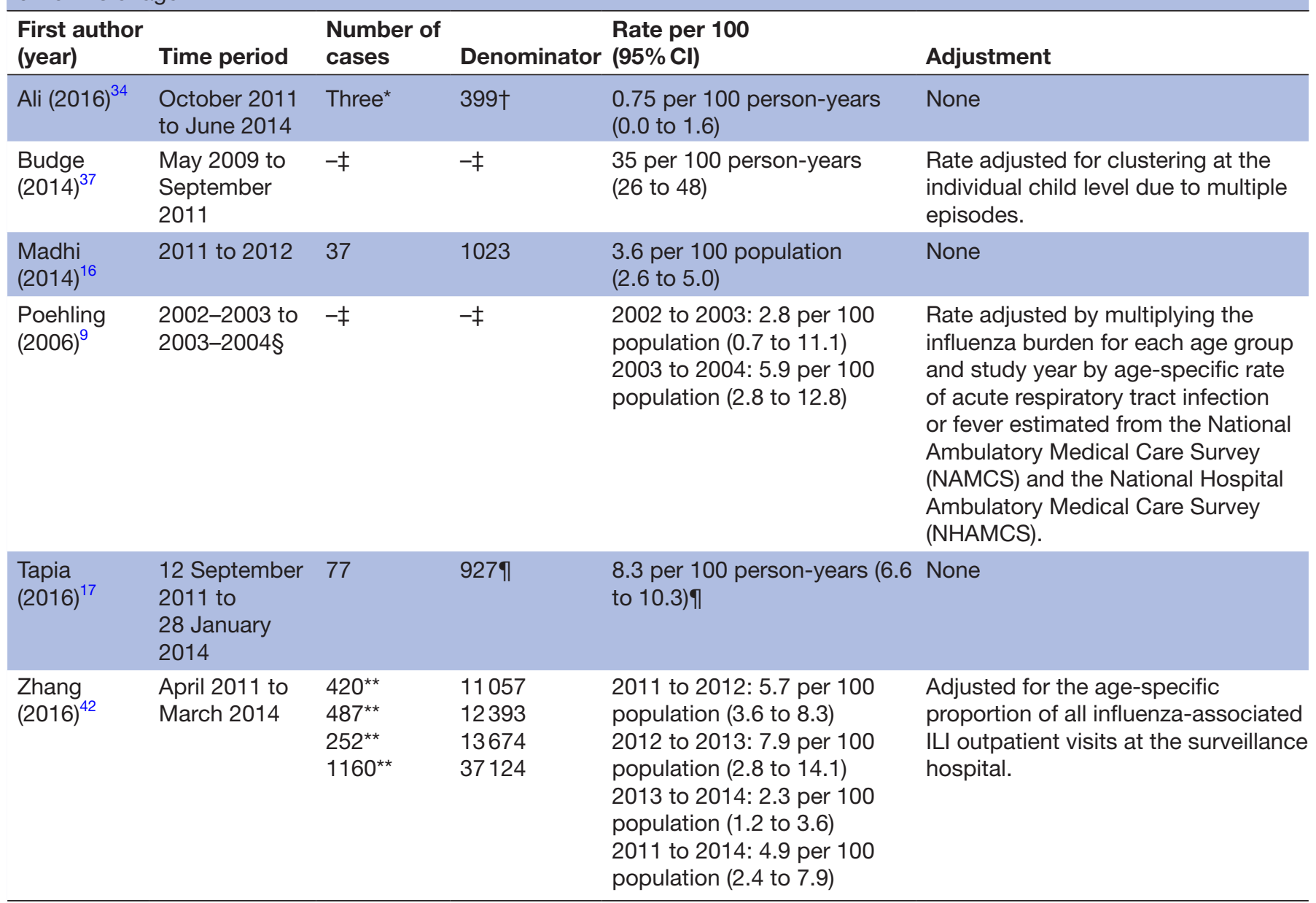

*Influenza B only.

†Number of person-years contributed by 692 infants.

$\ddagger$ Not reported in original study and insufficient information to compute.

§Rates based on number of outpatient clinic visits attributable to influenza in 2002-2003 to 2003-2004 only.

१Number of person-years contributed by 2041 infants. Rate and $95 \% \mathrm{Cl}$ derived by converting person-days to person-years.

${ }^{\star *}$ Age-specific numerators estimated by multiplying the number of visits for influenza-like illness (ILI) by the influenza positive proportion of enrolled ILI cases.

in a figure only. ${ }^{46}$ Estimated rates of LCI ICU admission for seasonal influenza ranged from a low of 0.5 per 10000 infants (95\% CI 0.8 to 16.5) between 2000-2001 and 2003-2004 in the Salt Lake City area of the USA ${ }^{9}$ to a high of 3.5 per 10000 between 2001 and 2004 in the surveillance counties covered by the NVSN (95\% CI 1.7 to 6.4$).{ }^{26}$ The absolute number of LCI ICU admissions of infants under 6 months was very low in all study populations (from a low of zero ${ }^{32}$ to a high of 12 admissions $^{40}$ ). Two studies were conducted during the $2009 \mathrm{H} 1 \mathrm{~N} 1$ pandemic time period-in Argentina, Libster et al reported a rate of LCI ICU admission of 2.9 per 10000 infants (95\% CI 1.6 to 5.0$)^{40}$ and in Israel, Stein $e$ al reported a similar rate of 2.5 per 10000 infants (95\% CI 0.79 to 6.0$).{ }^{46}$

\section{LCI death}

Nine studies included LCI death among infants under 6 months of age as an outcome. 71116172628354044 In six of the nine study populations, surveillance for LCI deaths was conducted, but none were identified ${ }^{11} 16263544$ (table 5). Bhat et al reported data from enhanced national-level surveillance of paediatric LCI deaths in the USA during the 2003-2004 season. ${ }^{7}$ In this study, there were 18 deaths of infants under 6 months of age, corresponding to a rate of 0.88 per 100000 infants $(95 \%$ CI 0.52 to 1.39 ), which was the highest among all paediatric age groups up to 18 years. In a smaller US surveillance study using data from the EIP system operating in 10 states, three influenza deaths of infants under 6 months were recorded during 2003-2004 to 2007-2008 combined, with a corresponding rate of 0.41 per 100000 personyears (95\% CI 0.11 to 1.12$).^{28}$ Among all nine studies, the highest rate of LCI deaths in infants was reported in Buenos Aires, Argentina, for the 2009 pandemic H1N1 time period; two deaths were recorded, and the LCI mortality rate was 5 per 100000 infants $(95 \%$ CI 0.82 to $16.1) .^{40}$ 


\begin{tabular}{ccc} 
Study & Time period & Country \\
McMorrow (2015) & 2008 & Kenya \\
McMorrow (2015) & 2011 & Kenya \\
McMorrow (2015) & 2012 & Kenya \\
Montes (2005) & $2002-2003$ & Spain \\
Ji (2010) & 2007 & China \\
Ji (2010) & 2008 & China \\
Dawood (2010) & $2006-2007$ & US \\
Madhi (2014) & $2011-2012$ & South Africa \\
Tapia (2016) & $2011-2014$ & Mali \\
Proff (2009) & $2006-2007$ & US \\
Cox (2012) & $2008-2009$ & US \\
Dawood (2010) & $2005-2006$ & US \\
Dawood (2010) & $2004-2005$ & US \\
Poehling (2013) & $2008-2009$ & US \\
Dawood (2010) & $2007-2008$ & US \\
Broor (2014) & $2009-2011$ & India \\
Proff (2009) & $2004-2005$ & US \\
Poehling (2013) & $2006-2007$ & US \\
Cox (2012) & $2009-2010$ & US \\
Libster (2010) & 2009 & Argentina \\
Proff (2009) & $2007-2008$ & US \\
Poehling (2006) & $2002-2003$ & US \\
Proff (2009) & $2005-2006$ & US \\
Iwane (2004) & $2000-2001$ & US \\
Poehling (2006) & $2000-2001$ & US \\
Poehling (2013) & $2005-2006$ & US \\
Ampofo (2006) & $2001-2004$ & US \\
Silvennoinen (2011) $1988-2004$ & Finland \\
Dawood (2010) & $2003-2004$ & US \\
Stein (2010) & 2009 & Israel \\
Poehling (2013) & $2004-2005$ & US \\
Griffin (2004) & $2000-2004$ & US \\
Poehling (2006) & $2001-2002$ & US \\
Poehling (2013) & $2007-2008$ & US \\
Cohen (2016) & $2010-2013$ & South Africa \\
Grijalva (2007) & $2004-2005$ & US \\
McMorrow (2015) & 2010 & Kenya \\
Montes (2005) & $2001-2002$ & Spain \\
Nelson (2014) & $2005-2011$ & Hong Kong \\
Poehling (2006) & $2003-2004$ & US \\
Montes (2005) & $2003-2004$ & Spain \\
Grijalva (2006) & $2003-2004$ & US \\
Bennet (2016) & $1998-2014$ & Sweden \\
Nelson (2014) & $2005-2011$ & Hong Kong \\
Yu (2014) & $2010-2012$ & China \\
McMorrow (2015) & 2009 & Kenya \\
\multicolumn{1}{c}{} & \\
Mcho & &
\end{tabular}

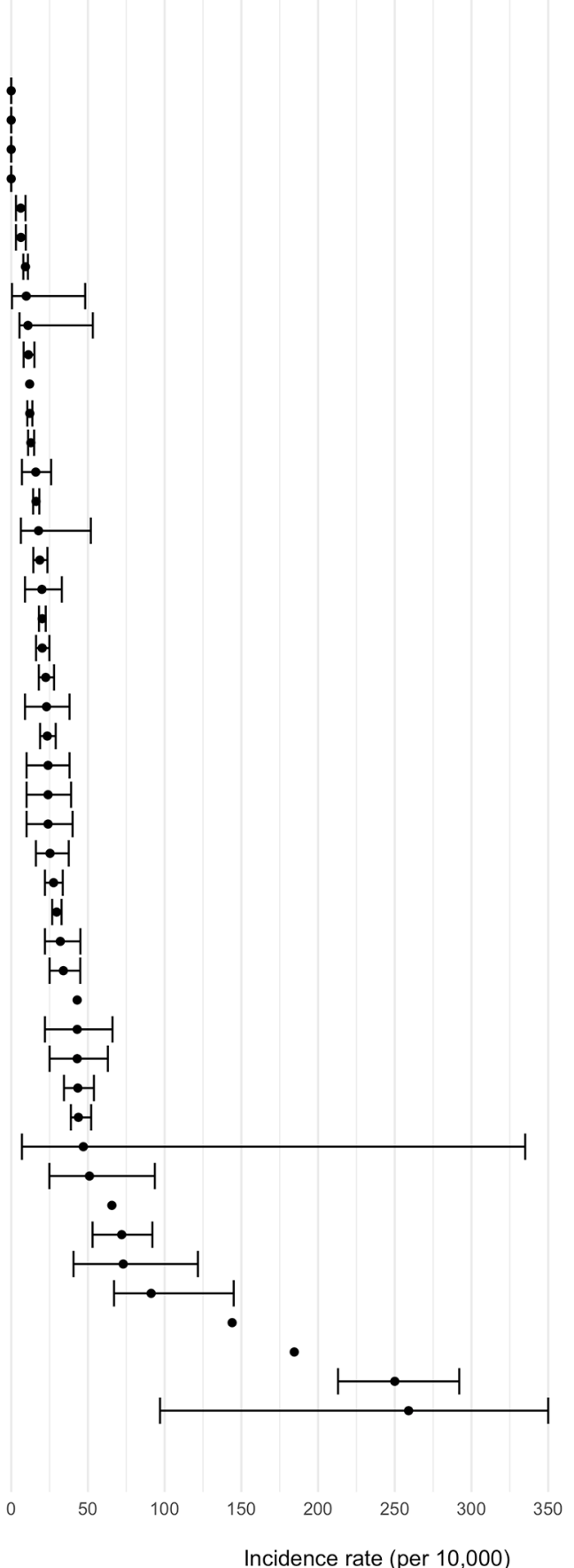

Figure 2 Incidence estimates of laboratory-confirmed influenza ( $\mathrm{LCl}$ ) hospitalisation among infants under 6 months of age. Heterogeneity 12: 100\%. All estimates can be found in online supplementary table S3.

\section{DISCUSSION}

In this systematic review, we provide a summary of published data up to April 2017 regarding the incidence of LCI outcomes among infants under 6 months of age. Our review of 27 studies covering 14 influenza seasons demonstrates a relatively wide range of estimates in incidence rates for several LCI outcomes in this age group. This broad distribution is likely associated with biological variability of influenza clinical disease and epidemiology and host immunity, as well as methodological factors of the studies themselves, including differences in care provider practices for influenza testing and hospital admission. In particular, estimates of rates for LCI hospitalisations, the most frequently reported and best-described outcome among these studies, ranged 10-fold, from 9.3 to 91.2 per 10000 infants, within the USA alone, and varied even more widely in other settings. The incidence of LCI hospitalisations was generally higher during the 2009 pandemic H1N1 time period (20 per 10000 infants ${ }^{40}$ to $259^{36}$ per 10000 person-years) than during seasonal influenza years, though few estimates from the H1N1 pandemic time period were available. Our work 
Table 3 Incidence estimates of laboratory-confirmed influenza hospitalisation among infants under 6 months of age from USbased influenza surveillance systems

\begin{tabular}{|c|c|c|c|}
\hline \multirow[b]{2}{*}{ Influenza season reported } & \multicolumn{3}{|l|}{ Surveillance system } \\
\hline & $\begin{array}{l}\text { EIP } \\
\text { Rate per } 10000 \text { population } \\
(95 \% \mathrm{Cl})\end{array}$ & $\begin{array}{l}\text { NVSN } \\
\text { Rate per } 10000 \text { population } \\
(95 \% \mathrm{Cl})\end{array}$ & $\begin{array}{l}\text { EIP and NVSN combined } \\
\text { Rate per } 10000 \text { population } \\
(95 \% \mathrm{Cl})\end{array}$ \\
\hline \multicolumn{4}{|l|}{ Individual seasons } \\
\hline 2000-2001 & & $\begin{array}{l}24.0(10.0 \text { to } 38.0)^{32} \\
24.0(10.0 \text { to } 39.0)^{9}\end{array}$ & \\
\hline 2001-2002 & & $43.0(22.0 \text { to } 66.0)^{9}$ & \\
\hline 2002-2003 & & $23.0(9.0 \text { to } 38.0)^{9}$ & \\
\hline 2003-2004 & $29.6(26.7 \text { to } 32.8)^{28}$ & $72.0(53.0 \text { to } 92.0)^{9}$ & $91.2(67.0 \text { to } 145.0)^{* 30}$ \\
\hline 2004-2005 & $12.8(11.0 \text { to } 14.9)^{28}$ & $34.0(25.0 \text { to } 45.0)^{8}$ & $43.8(38.9$ to 52.1$) \dagger^{31}$ \\
\hline 2005-2006 & $12.1(10.5 \text { to } 13.8)^{28}$ & $24.0(10.0 \text { to } 40.0)^{8}$ & \\
\hline 2006-2007 & $9.3(7.9 \text { to } 10.9)^{28}$ & $20.0(9.0 \text { to } 33.0)^{8}$ & \\
\hline 2007-2008 & $16.2(14.3 \text { to } 18.3)^{28}$ & $43.0(25.0 \text { to } 63.0)^{8}$ & \\
\hline 2008-2009 & $12.0^{27}$ & $16.0(7.0 \text { to } 26.0)^{8}$ & \\
\hline 2009-2010 (H1N1 pandemic) & $20.2(18.1 \text { to } 22.5)^{27}$ & & \\
\hline \multicolumn{4}{|l|}{ Combined seasons } \\
\hline 2000-2004 & & $\begin{array}{l}43.0^{29} \\
45.0(34.0 \text { to } 55.0)^{9}\end{array}$ & \\
\hline 2004-2009 & & $27.0(21.0 \text { to } 33.0)^{9}$ & \\
\hline
\end{tabular}

*Individual estimates: $\mathrm{EIP}=34.5$ per 10,000; NVSN=66.6 per 10000.

†Individual estimates: EIP=17.4 per 10,000; NVSN=29.9 per 10000.

EIP, Emerging Infections Program; NVSN, New Vaccine Surveillance Network.

also highlights the relative lack of studies that specifically report influenza outcomes in this vulnerable age group and the limited information included in studies that do include such findings. LCI outcomes other than hospitalisation, such as ICU admission and death, were even less commonly assessed and varied markedly in the level of detail described. Moreover, the majority of data identified in this review come from the USA, deriving primarily from just two influenza surveillance systems, indicating the constrained geographic coverage of the collected datasets. These limitations pose challenges for estimating the potential impact of maternal influenza immunisation programmes on infant influenza outcomes, particularly for low- and middle-income countries.

\begin{tabular}{|c|c|c|c|c|c|}
\hline First author (year) & Time period & $\begin{array}{l}\text { Number of } \\
\text { cases }\end{array}$ & Denominator & $\begin{array}{l}\text { Rate per } 10000 \\
(95 \% \mathrm{Cl})\end{array}$ & Adjustment \\
\hline Ampofo $(2006)^{26}$ & July 2001 to June 2004 & Nine $^{*}$ & 25710 & $\begin{array}{l}3.5 \text { per } 10000 \text { population } \\
(1.7 \text { to } 6.4)^{\star}\end{array}$ & None \\
\hline Iwane $(20004)^{32}$ & 2000-2001 & 0 & 8591 & 0 & None \\
\hline Ji $(2010)^{39}$ & $\begin{array}{l}\text { January } 2007 \text { to } \\
\text { December } 2008\end{array}$ & 3 & $48147^{\star}$ & $\begin{array}{l}0.62 \text { per } 10000 \text { person-years } \\
(1.6 \text { to } 17.0)^{*}\end{array}$ & None \\
\hline Libster $(2010)^{40}$ & May 2009 to July 2009 & 12 & 41000 & $\begin{array}{l}2.9 \text { per } 10000 \text { population } \\
(1.6 \text { to } 5.0)^{\star}\end{array}$ & None \\
\hline Poehling $(2006)^{9}$ & $2000-2001$ to $2003-2004$ & 2 & 40000 & $\begin{array}{l}0.5 \text { per } 10000 \text { population } \\
(0.8 \text { to } 16.5)^{\star}\end{array}$ & None \\
\hline Silvennoinen $(2011)^{45}$ & July 1988 to June 2004 & 5 & 31884 & $\begin{array}{l}1.6 \text { per } 10000 \text { population } \\
0.6 \text { to } 3.5)^{\star}\end{array}$ & None \\
\hline Stein $(2010)^{46}$ & $\begin{array}{l}12 \text { July } 2009 \text { to } \\
24 \text { December } 2009\end{array}$ & 4 & $16000^{*}$ & 2.5 per 10000 population $\dagger$ & None \\
\hline
\end{tabular}

*Estimated by review authors from information reported in original study. †Estimated from figure 2 in original study. ${ }^{46}$ 
Table 5 Incidence estimates of laboratory-confirmed influenza death among infants under 6 months of age

\begin{tabular}{|c|c|c|c|c|c|}
\hline First author (year) & Time period & $\begin{array}{l}\text { Number of } \\
\text { cases }\end{array}$ & Denominator & $\begin{array}{l}\text { Rate per } 100000 \\
(95 \% \mathrm{Cl})\end{array}$ & Adjustment \\
\hline Ampofo $(2006)^{26}$ & July 2001 to June 2004 & 0 & 25710 & 0 & None \\
\hline Broor $(2014)^{35}$ & August 2009 to July 2011 & 0 & $-^{*}$ & 0 & None \\
\hline Dawood $(2010)^{28}$ & $2003-2004$ to $2007-2008$ & 3 & $726886 \dagger$ & $\begin{array}{l}0.41 \text { per } 100000 \text { person- } \\
\text { years }(0.11 \text { to } 1.12) \dagger\end{array}$ & None \\
\hline Libster $(2010)^{40}$ & May 2009 to July 2009 & 2 & 41000 & $\begin{array}{l}\text { five per } 100000 \text { population } \\
(0.82 \text { to } 16.1) \dagger\end{array}$ & None \\
\hline Madhi $(2014)^{16}$ & 2011 to 2012 & 0 & 1023 & 0 & None \\
\hline Montes $(2005)^{11}$ & July 2001 to June 2004 & 0 & 5366 & 0 & None \\
\hline Nelson $(2014)^{44}$ & April 2005 to March 2011 & 0 & $-^{\star}$ & 0 & None \\
\hline
\end{tabular}

*Not reported in original study and insufficient information to compute. †Estimated by review authors from information reported in original study. $\ddagger$ Number of person-years contributed by 2041 infants.

Our review methodology used a comprehensive search strategy that emphasised high sensitivity to capture a broad set of articles for screening. We subsequently restricted our review to laboratory-confirmed, population-based estimates of influenza incidence, ensuring greater consistency and stability of rates across studies. Our review also benefits from a number of strengths regarding the original studies identified through our search. First, the majority of the data come from well-established surveillance systems that cover several seasons and include additional evaluations (eg, capture-recapture methods ${ }^{30}$ ) to confirm the validity of their findings. This consistency adds to the stability of the range of estimates reported here and provides a better understanding of the effects of seasonal variation on annual burden estimates. Second, the included studies generally obtained high scores in the quality assessment tool providing some assurance that they met at least minimum quality criteria.

Nevertheless, there are several important limitations. Among the primary studies, the different surveillance methodologies (eg, passive versus active surveillance; different sensitivity of diagnostic tests; recruitment only in a subset of days per week; different denominators or methods to calculate incidence rates; surveillance only during part of the year; rate adjustment for various factors) contributed to the heterogeneity of the results. Moreover, some studies reported only a small number of seasons or a limited geographic area which may not provide a fully representative assessment of typical influenza incidence. Although none of the non-randomised studies specifically reported the uptake of maternal influenza immunisation in their study population, the majority were either studies from the USA during pre-2009 pandemic seasons when rates were low, ${ }^{48}$ or were from settings without recommendations for influenza immunisation during pregnancy; consequently, this was unlikely to be an important contributor to the heterogeneity in incidence rates. We were unable to include several studies that aggregated data from infants under 6 months within larger age strata, thus not reporting data specific to this policy-relevant age group. Future surveillance studies should report data for infants under 6 months, even if only as supplementary data, to facilitate future pooling and meta-analyses. The reports themselves were incomplete at times, lacking numerator data, denominator data or precision estimates, precluding the ability to perform meta-analysis. Even with full reporting of the data, key factors may have influenced the accuracy and completeness of specific surveillance approaches. Importantly, Grijalva $e t a l^{3031}$ demonstrated that the two US surveillance systems each underestimate the incidence of LCI hospitalisations. In both 2003-2004 and 2004-2005, the incidence of LCI hospitalisation was higher using the combined capture-recapture methodology ${ }^{47}$ than when estimated using either NVSN or EIP data alone. Finally, several of the US-based studies described overlapping seasons across multiple reports, thus contributing to a risk of some duplicate reporting.

Although we evaluated studies that included a narrower age range compared with the review by Nair et al, ${ }^{1}$ our results are generally consistent with the findings presented in that review, which reported rates of severe acute lower respiratory infection (corresponding to influenza hospitalisation) ranging from 10 to 170 per 10000 person-years among infants under 1 year of age. These consistent findings support the overall interpretation that influenza has a significant role in early infant respiratory morbidity. Of note, the incidence rates reported for influenza hospitalisation in the control arms of the randomised clinical trials included in this review ${ }^{16}{ }^{17}$ were at the lower end of 
the range of estimates (one infant LCI hospitalisation in each trial, corresponding to a rate of 9.8 per 10000 infants in South Africa (personal communication: M Nunes, 7 December 2016), and 10.8 per 10000 person-years in Mali [personal communication: M Tapia, 15 Dec 2016]). These low rates compared with other estimates could be due to the epidemiological characteristics of the particular influenza seasons or due to the close observation of subjects and opportunity for treatment and follow-up afforded by the active surveillance in the trials. Using prospective active surveillance methods, these two trials likely provide the best estimates of the incidence of LCI illness in an ambulatory setting among infants under 6 months: 3.6 per 100 infants ${ }^{16}$ and 8.3 per 100 person-years of follow-up. ${ }^{17}$ Another recent trial of maternal influenza immunisation in Nepal, published subsequent to our literature search, documented an incidence rate of LCI illness in an ambulatory setting of 18.1 per 100 person-years of follow-up among infants in the placebo group. ${ }^{49}$

In conclusion, our systematic review demonstrates that existing data on LCI outcomes among infants under 6 months of age are sparse, of varying quality, and heavily weighted towards high-income populations. More research is needed in key regions to obtain a more globally representative picture of the incidence of influenza outcomes among young infants. In particular, estimates are required from the low-income and low-middle-income countries of Asia and Africa where, in absolute numbers, the majority of cases occur. Higher quality data will be essential in order to allow global and country-level policymakers to make evidence-based decisions that appropriately prioritise interventions, such as maternal influenza immunisation for reducing influenza disease in young infants who are, themselves, not eligible for influenza vaccination.

\author{
Author affiliations \\ ${ }^{1}$ University of Ottawa and Children's Hospital of Eastern Ontario (CHEO) Research \\ Institute, Ottawa, Ontario, Canada \\ ${ }^{2}$ Independent Epidemiology Consultant, Los Gatos, California, USA \\ ${ }^{3}$ Tel Aviv Department of Health, Ministry of Health, Tel Aviv, Israel \\ ${ }^{4}$ Sackler School of Medicine, Tel Aviv University, Tel Aviv, Israel \\ ${ }^{5}$ Medical School for International Health and Department of Health Systems \\ Management, Ben Gurion University in the Negev, Beersheba, Israel \\ ${ }^{6}$ Department of Epidemiology, School of Public Health, University of Michigan, Ann \\ Arbor, Michigan, USA \\ ${ }^{7}$ Independent Information Specialist, Ottawa, Ontario, Canada \\ ${ }^{8}$ Department of Medicine, Center for Vaccine Development, University of Maryland \\ School of Medicine, Baltimore, Maryland, USA \\ ${ }^{9}$ Initiative for Vaccine Research, WHO, Geneva, Switzerland \\ ${ }^{10}$ Center for Vaccine Innovation and Access, PATH, Seattle, Washington, USA
}

Acknowledgements We acknowledge members of the WHO Taskforce to Evaluate Influenza Data to Inform Vaccine Impact and Economic Modelling, a working group of the WHO Initiative for Vaccine Research, for their contributions through early discussions about this study. We are grateful to Glen Zinck (PATH) for his help with project organisation, to Dr. Corinne Riddell (McGill University) for her assistance with producing Forest plots and to Laura Walsh (University of Ottawa) for her assistance with updating the data extraction. We additionally thank Dr. Kevin Pottie (University of Ottawa), Dr. Michael Gravett (University of Washington) and Dr. Dayre McNally (University of Ottawa) for their thoughtful reviews of an earlier version of this manuscript.
Contributors NB, KMN and JRO designed the study protocol. BS designed and ran the systematic search strategy. JJ, ZM and MAK screened the articles, extracted the data and assessed the quality of individual studies. DBF, ZM, MAK and JRO contacted study authors for additional information. DBF interpreted the data and wrote the first draft of the manuscript. All authors critically revised the manuscript for intellectual content, gave final approval of the version to be published and agreed to be accountable for all aspects of the work.

Funding This work was supported by the Bill \& Melinda Gates Foundation through the WHO. The research was coordinated by PATH. DBF and MAK received financial support from the WHO's Initiative for Vaccine Research. The authors also acknowledge the Centers for Disease Control and Prevention, which provides financial support to the WHO's Initiative for Vaccine Research (U50 CK000431).

Disclaimer JRO is an employee of the WHO. The authors alone are responsible for the views expressed in this publication and they do not necessarily represent the decisions, policy or views of the WHO.

Competing interests None declared.

Provenance and peer review Not commissioned; externally peer reviewed.

Data sharing statement The data set is available on request from the corresponding author.

Open Access This is an Open Access article distributed in accordance with the Creative Commons Attribution Non Commercial (CC BY-NC 4.0) license, which permits others to distribute, remix, adapt, build upon this work non-commercially, and license their derivative works on different terms, provided the original work is properly cited and the use is non-commercial. See: http://creativecommons.org/ licenses/by-nc/4.0/

(C) Article author(s) (or their employer(s) unless otherwise stated in the text of the article) 2017. All rights reserved. No commercial use is permitted unless otherwise expressly granted.

\section{REFERENCES}

1. Nair H, Brooks WA, Katz M, et al. Global burden of respiratory infections due to seasonal influenza in young children: a systematic review and meta-analysis. Lancet 2011;378:1917-30.

2. Lafond KE, Nair H, Rasooly $\mathrm{MH}$, et al. Global role and burden of Influenza in pediatric respiratory hospitalizations, 1982-2012: a systematic analysis. PLoS Med 2016;13:e1001977.

3. Lozano R, Naghavi M, Foreman K, et al. Global and regional mortality from 235 causes of death for 20 age groups in 1990 and 2010: a systematic analysis for the global burden of disease study 2010. Lancet 2012;380:2095-128.

4. Munoz FM. Influenza virus infection in infancy and early childhood. Paediatr Respir Rev 2003;4:99-104.

5. Moore DL, Vaudry W, Scheifele DW, et al. Surveillance for influenza admissions among children hospitalized in Canadian immunization monitoring program active centers, 2003-2004. Pediatrics 2006;118:e610-e619.

6. Smith NM, Bresee JS, Shay DK, et al. Prevention and Control of Influenza: recommendations of the Advisory Committee on Immunization Practices (ACIP). MMWR Recomm Rep 2006;55(RR10):1-42.

7. Bhat N, Wright JG, Broder KR, et al. Influenza-associated deaths among children in the United States, 2003-2004. N Engl J Med 2005;353:2559-67.

8. Poehling KA, Edwards KM, Griffin MR, et al. The burden of influenza in young children, 2004-2009. Pediatrics 2013;131:207-16.

9. Poehling KA, Edwards KM, Weinberg GA, et al. The underrecognized burden of influenza. N Engl J Med 2006;355:31-40.

10. Neuzil KM, Mellen BG, Wright PF, et al. The effect of influenza on hospitalizations, outpatient visits, and courses of antibiotics in children. N Engl J Med 2000;342:225-31.

11. Montes M, Vicente D, Pérez-Yarza EG, et al. Influenza-related hospitalisations among children aged less than 5 years old in the Basque Country, Spain: a 3-year study (July 2001-June 2004). Vaccine 2005;23:4302-6.

12. Izurieta HS, Thompson WW, Kramarz P, et al. Influenza and the rates of hospitalization for respiratory disease among infants and young children. N Engl J Med 2000;342:232-9.

13. Fiore AE, Uyeki TM, Broder K, et al. Prevention and control of influenza with vaccines: recommendations of the Advisory Committee on Immunization Practices (ACIP), 2010. MMWR Recomm Rep 2010;59(RR-8):1-62. 
14. Ortiz JR, Neuzil KM. The promise of maternal vaccination to prevent influenza in young infants. Arch Pediatr Adolesc Med 2011;165:179-80.

15. Zaman K, Roy E, Arifeen SE, et al. Effectiveness of maternal influenza immunization in mothers and infants. $N$ Engl $\mathrm{J} \mathrm{Med}$ 2008;359:1555-64.

16. Madhi SA, Cutland CL, Kuwanda L, et al. Influenza vaccination of pregnant women and protection of their infants. N Engl J Med 2014;371:918-31.

17. Tapia MD, Sow SO, Tamboura B, et al. Maternal immunisation with trivalent inactivated influenza vaccine for prevention of influenza in infants in Mali: a prospective, active-controlled, observer-blind, randomised phase 4 trial. Lancet Infect Dis 2016;16:1026-35.

18. World Health Organization. WHO Taskforce to evaluate Influenza Data to inform Vaccine Impact and Economic Modelling. Geneva, Switzerland, 2014. http://www.who.int/immunization/research/meet ings_workshops/taskforceinterimreportMarch2015/en/. (accessed 20 feb 2017).

19. Moher D, Liberati A, Tetzlaff J, et al. Preferred reporting items for systematic reviews and meta-analyses: the PRISMA statement. BMJ 2009;339:b2535.

20. Hang LK, Do LP, Van TT, et al. Viral co-infections among children with confirmed measles at hospitals in Hanoi, Vietnam, 2014. Asian Pac J Trop Med 2017;10:171-4.

21. Munn Z, Moola S, Riitano D, et al. The development of a critical appraisal tool for use in systematic reviews addressing questions of prevalence. Int J Health Policy Manag 2014;3:123-8.

22. Guyatt G, Oxman AD, Akl EA, et al. GRADE guidelines: 1. Introduction-GRADE evidence profiles and summary of findings tables. J Clin Epidemiol 2011;64:383-94.

23. Huguet A, Hayden JA, Stinson J, et al. Judging the quality of evidence in reviews of prognostic factor research: adapting the GRADE framework. Syst Rev 2013;2:71.

24. Deeks JJ, Higgins JPT, Altman DG. on behalf of the Cochrane Statistical Methods GroupThe Cochrane Collaboration. Analysing data and undertaking meta-analyses. In: Higgins JPT, Green S, eds. Cochrane Handbook for Systematic Reviews of Interventions. Version 5.1.0 (updated March 2011, 2011:9.1-9.43. http://www. cochrane.org/training/cochrane-handbook. (accessed 20 feb 2017).

25. Higgins JP, Thompson SG. Quantifying heterogeneity in a metaanalysis. Stat Med 2002;21:1539-58.

26. Ampofo K, Gesteland PH, Bender J, et al. Epidemiology, complications, and cost of hospitalization in children with laboratoryconfirmed influenza infection. Pediatrics 2006;118:2409-17.

27. Cox CM, D'Mello T, Perez A, et al. Increase in rates of hospitalization due to laboratory-confirmed influenza among children and adults during the 2009-10 influenza pandemic. J Infect Dis 2012;206:1350-8.

28. Dawood FS, Fiore A, Kamimoto L, et al. Burden of seasonal influenza hospitalization in children, United States, 2003 to 2008. J Pediatr 2010;157:808-14.

29. Griffin MR, Walker FJ, Iwane MK, et al. Epidemiology of respiratory infections in young children. Pediatr Infect Dis J 2004;23:S188-S192.

30. Grijalva CG, Craig AS, Dupont WD, et al. Estimating influenza hospitalizations among children. Emerg Infect Dis 2006;12:103-9.

31. Grijalva CG, Weinberg GA, Bennett NM, et al. Estimating the undetected burden of influenza hospitalizations in children. Epidemiol Infect 2007;135:951-8.
32. Iwane MK, Edwards KM, Szilagyi PG, et al. Population-based surveillance for hospitalizations associated with respiratory syncytial virus, influenza virus, and parainfluenza viruses among young children. Pediatrics 2004;113:1758-64.

33. Proff R, Gershman K, Lezotte D, et al. Case-based surveillance of influenza hospitalizations during 2004-2008, Colorado, USA. Emerg Infect Dis 2009;15:892-8.

34. Ali A, Akhund T, Warraich GJ, et al. Respiratory viruses associated with severe pneumonia in children under 2 years old in a rural community in Pakistan. J Med Virol 2016;88:1882-90.

35. Broor S, Dawood FS, Pandey BG, et al. Rates of respiratory virusassociated hospitalization in children aged $<5$ years in rural northern India. J Infect 2014;68:281-9.

36. McMorrow ML, Emukule GO, Njuguna HN, et al. The unrecognized burden of Influenza in young Kenyan children, 2008-2012. PLoS One 2015;10:e0138272-12

37. Budge PJ, Griffin MR, Edwards KM, et al. A household-based study of acute viral respiratory illnesses in Andean children. Pediatr Infect Dis J 2014;33:443-7.

38. Cohen C, Moyes J, Tempia S, et al. Epidemiology of acute lower respiratory tract infection in HIV-exposed uninfected infants. Pediatrics 2016;137:e20153272.

39. Ji W, Zhang T, Zhang X, et al. The epidemiology of hospitalized influenza in children, a two year population-based study in the People's Republic of China. BMC Health Serv Res 2010;10:82.

40. Libster R, Bugna J, Coviello S, et al. Pediatric hospitalizations associated with 2009 pandemic influenza A (H1N1) in Argentina. N Engl J Med 2010;362:45-55.

41. Yu H, Huang J, Huai Y, et al. The substantial hospitalization burden of influenza in central China: surveillance for severe, acute respiratory infection, and influenza viruses, 2010-2012. Influenza Other Respir Viruses 2014;8:53-65.

42. Zhang T, Zhang J, Hua J, et al. Influenza-associated outpatient visits among children less than 5 years of age in eastern China, 20112014. BMC Infect Dis 2016;16:267

43. Bennet R, Hamrin J, Wirgart BZ, et al. Influenza epidemiology among hospitalized children in Stockholm, Sweden 1998-2014. Vaccine 2016;34:3298-302

44. Nelson EA, Ip M, Tam JS, et al. Burden of influenza infection in hospitalised children below 6 months of age and above in Hong Kong from 2005 to 2011. Vaccine 2014;32:6692-8.

45. Silvennoinen $\mathrm{H}$, Peltola $\mathrm{V}$, Vainionpää $\mathrm{R}$, et al. Incidence of influenzarelated hospitalizations in different age groups of children in Finland: a 16-year study. Pediatr Infect Dis J 2011;30:e24-e28.

46. Stein M, Tasher D, Glikman D, et al. Hospitalization of children with influenza $A(H 1 N 1)$ virus in Israel during the 2009 outbreak in Israel: a multicenter survey. Arch Pediatr Adolesc Med 2010;164:1015-22.

47. Hook EB, Regal RR. Capture-recapture methods in epidemiology: methods and limitations. Epidemiol Rev 1995;17:243-64.

48. Groom HC, Henninger ML, Smith N, et al. Influenza vaccination during pregnancy: influenza seasons 2002-2012, Vaccine Safety Datalink. Am J Prev Med 2016;50:480-8.

49. Steinhoff MC, Katz J, Englund JA, et al. Year-round influenza immunisation during pregnancy in Nepal: a phase 4, randomised, placebo-controlled trial. Lancet Infect Dis 2017. 
Correction: Incidence of laboratory-confirmed influenza

disease among infants under 6 months of age: $a$

systematic review

Fell DB, Johnson J, Mor Z, et al. Incidence of laboratory-confirmed influenza disease among infants under 6 months of age: a systematic review. BMJ Open 2017;7:e016526. doi: 10.1136/bmjopen-2017-016526

In figure 2 and online supplementary table 3 , Bennet (2016) are reported to have found an influenza incidence of 144 per 10000 in infants younger than six months; however, the correct figure is a median yearly incidence $1998-2014$ of 144 per $10^{5}$ in this age group, which equals 14.4 per 10000 . This correct figure is among the lowest in similar settings, and considerably reduces the variability of reported incidences.

The corrected figure 2 is shown below:
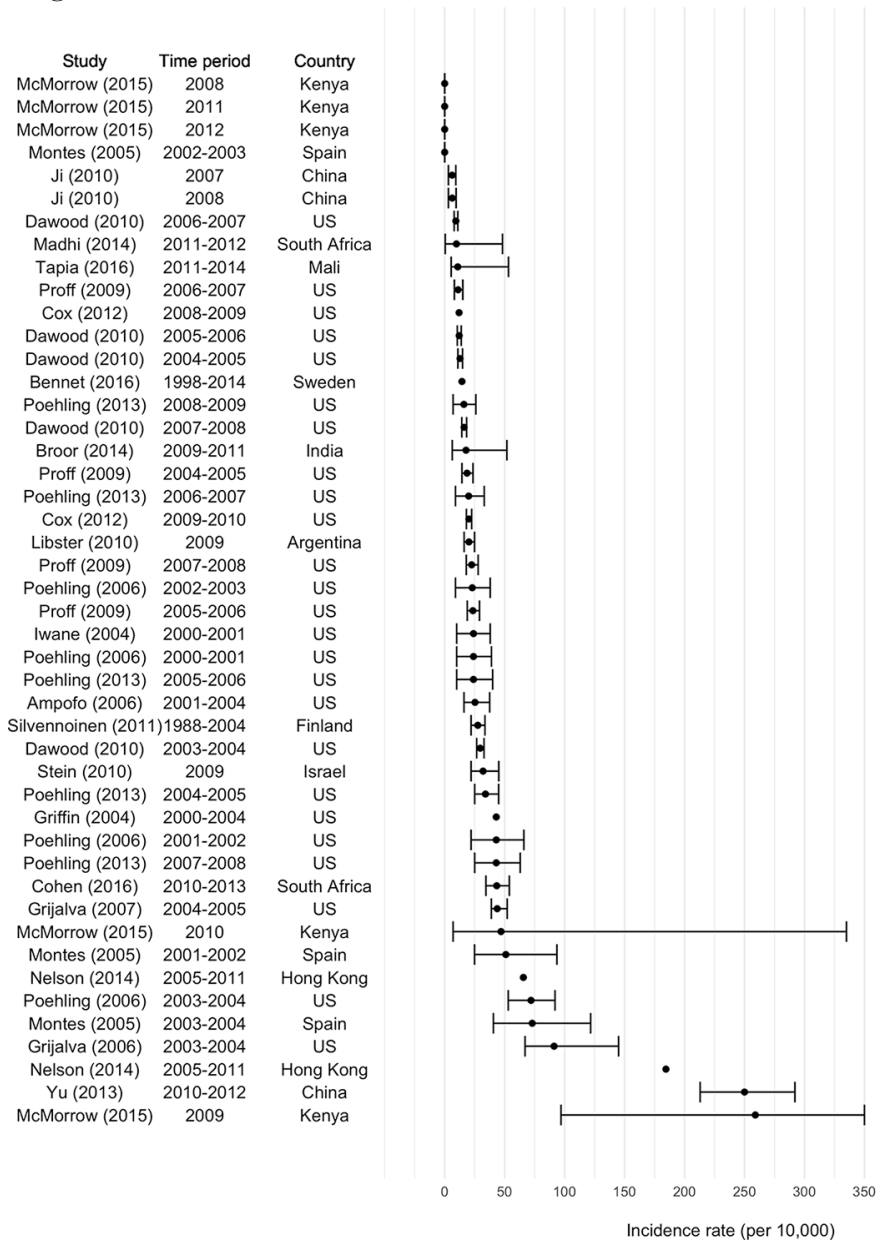

A corrected version of the supplementary table is available here: revised supplementary table 3 .

Open Access This is an Open Access article distributed in accordance with the Creative Commons Attribution Non Commercial (CC BY-NC 4.0) license, which permits others to distribute, remix, adapt, build upon this work non-commercially, and license their derivative works on different terms, provided the original work is properly cited and the use is non-commercial. See: http://creativecommons.org/licenses/by-nc/4.0/

(C) Article author(s) (or their employer(s) unless otherwise stated in the text of the article) 2018. All rights reserved. No commercial use is permitted unless otherwise expressly granted.

BMJ Open 2018;8:e016526corr1. doi:10.1136/bmjopen-2017-016526corr1

Check for updates 